Irish Math. Soc. Bulletin

Number 82, Winter 2018, 39-43

ISSN 0791-5578

\title{
A duplication theorem for the Hermite polynomials
}

\author{
HORST ALZER
}

ABstract. The generalized Hermite polynomials $H_{n, p}(z)$ are generated by

$$
\exp \left(p z t-t^{p}\right)=\sum_{n=0}^{\infty} \frac{H_{n, p}(z)}{n !} t^{n} \quad(p \in \mathbb{N}) .
$$

We prove that the formula

$$
H_{n, p}(a z)=n ! \sum_{k=0}^{[n / p]} \frac{\left(a^{p}-1\right)^{k} a^{n-p k}}{k !(n-p k) !} H_{n-p k, p}(z)
$$

holds for all integers $n \geq 0, p \geq 1$ and $a, z \in \mathbb{C}$. The special case $p=a=2$ leads to the following duplication theorem for the classical Hermite polynomials:

$$
H_{n}(2 z)=n ! 2^{n} \sum_{k=0}^{[n / 2]}\left(\frac{3}{4}\right)^{k} \frac{H_{n-2 k}(z)}{k !(n-2 k) !} .
$$

The classical Hermite polynomials $H_{n}(z)(n=0,1,2, \ldots ; z \in \mathbb{C})$, named after the French mathematician Charles Hermite (1822 1901), are generated by

$$
\exp \left(2 z t-t^{2}\right)=\sum_{n=0}^{\infty} \frac{H_{n}(z)}{n !} t^{n}
$$

They can be written explicitly as

$$
H_{n}(z)=n ! \sum_{k=0}^{[n / 2]}(-1)^{k} \frac{2^{n-2 k}}{k !(n-2 k) !} z^{n-2 k}
$$

2010 Mathematics Subject Classification. 33C45.

Key words and phrases. Hermite polynomials, generalized Hermite polynomials, duplication theorem.

Received on 3-10-2018; revised 13-11-2018. 
or by using the Rodrigues formula as

$$
H_{n}(z)=(-1)^{n} e^{z^{2}} \frac{d^{n}}{d z^{n}} e^{-z^{2}}
$$

In particular, $H_{n}$ is a polynomial of degree $n$ with leading coefficient $2^{n}$. The first few polynomials are

$$
\begin{gathered}
H_{0}(z)=1, \quad H_{1}(z)=2 z, \quad H_{2}(z)=4 z^{2}-2, \\
H_{3}(z)=8 z^{3}-12 z, \quad H_{4}(z)=16 z^{4}-48 z^{2}+12 .
\end{gathered}
$$

We have the symmetry relation

$$
H_{n}(-z)=(-1)^{n} H_{n}(z)
$$

the recurrence relations

$$
H_{n+1}(z)=2 z H_{n}(z)-2 n H_{n-1}(z), \quad H_{n}^{\prime}(z)=2 n H_{n-1}(z)
$$

and the pseudo-addition formula

$$
H_{n}(x+y)=2^{-n / 2} \sum_{k=0}^{n}\left(\begin{array}{l}
n \\
k
\end{array}\right) H_{k}(\sqrt{2} x) H_{n-k}(\sqrt{2} y) .
$$

In the theory of differential equations $H_{n}$ appears as a solution of the second-order linear differential equation

$$
y^{\prime \prime}-2 x y^{\prime}+2 n y=0 .
$$

There is a large body of literature on these functions. Indeed, since the Hermite polynomials have remarkable applications in the theory of special functions, probability theory, physics and other fields, they have attracted the attention of numerous researchers. Their main properties are collected, for example, in [1, chapter 22], [2, chapter 13.1], [5, chapter 1].

In this note, we present a duplication theorem for the Hermite polynomials which we could not locate in the literature.

Theorem 1. For all nonnegative integers $n$ and complex numbers $z$ we have

$$
H_{n}(2 z)=n ! 2^{n} \sum_{k=0}^{[n / 2]}\left(\frac{3}{4}\right)^{k} \frac{H_{n-2 k}(z)}{k !(n-2 k) !} .
$$


Actually, we prove a bit more. We offer an identity satisfied by the generalized Hermite polynomials $H_{n, p}(z)$ which are given by

$$
\exp \left(p z t-t^{p}\right)=\sum_{n=0}^{\infty} \frac{H_{n, p}(z)}{n !} t^{n} \quad(p \in \mathbb{N}) .
$$

Obviously, $H_{n, 2}=H_{n}$. We have the explicit representation

$$
H_{n, p}(z)=n ! \sum_{k=0}^{[n / p]}(-1)^{k} \frac{p^{n-p k}}{k !(n-p k) !} z^{n-p k} .
$$

See [3] and [4] for more information on these functions.

We show that the following extension of (1) is valid.

Theorem 2. For all integers $n \geq 0, p \geq 1$ and complex numbers a, $z$ we have

$$
H_{n, p}(a z)=n ! \sum_{k=0}^{[n / p]} \frac{\left(a^{p}-1\right)^{k} a^{n-p k}}{k !(n-p k) !} H_{n-p k, p}(z) .
$$

Proof We have

$$
\exp \left(p \cdot a z \cdot t-t^{p}\right)=\exp \left(p \cdot z \cdot a t-(a t)^{p}\right) \cdot \exp \left(\left(a^{p}-1\right) t^{p}\right) .
$$

From (2) and (4) we obtain

$$
\sum_{n=0}^{\infty} \frac{H_{n, p}(a z)}{n !} t^{n}=\sum_{n=0}^{\infty} \frac{a^{n} H_{n, p}(z)}{n !} t^{n} \cdot \sum_{n=0}^{\infty} \frac{\left(a^{p}-1\right)^{n}}{n !} t^{p n} .
$$

Let

$$
u_{n}=u_{n, p}(a, z)=\frac{a^{n} H_{n, p}(z)}{n !}, \quad v_{n}=v_{n, p}(a)=\frac{\left(a^{p}-1\right)^{n}}{n !}
$$

and

$$
\delta_{n}=\delta_{n, p}= \begin{cases}v_{n / p}, & \text { if } p \mid n \\ 0, & \text { otherwise }\end{cases}
$$

We have

$$
\sum_{n=0}^{\infty} v_{n} t^{p n}=\sum_{n=0}^{\infty} \delta_{n} t^{n}
$$


Applying (6) yields

$$
\begin{aligned}
\sum_{n=0}^{\infty} u_{n} t^{n} \cdot \sum_{n=0}^{\infty} v_{n} t^{p n} & =\sum_{n=0}^{\infty} \sum_{\nu=0}^{n} u_{n-\nu} \delta_{\nu} t^{n} \\
& =\sum_{n=0}^{\infty} \sum_{k=0}^{[n / p]} u_{n-p k} \delta_{p k} t^{n} \\
& =\sum_{n=0}^{\infty} \sum_{k=0}^{[n / p]} u_{n-p k} v_{k} t^{n}
\end{aligned}
$$

A comparison of the coefficients of the power series given in (5) and (7) reveals that

$$
\frac{H_{n, p}(a z)}{n !}=\sum_{k=0}^{[n / p]} u_{n-p k} v_{k}=\sum_{k=0}^{[n / p]} \frac{a^{n-p k} H_{n-p k, p}(z)}{(n-p k) !} \frac{\left(a^{p}-1\right)^{k}}{k !}
$$

which is $(3)$.

Remark 1. If we set $p=a=2$ in (3), then we obtain the duplication formula (1).

Remark 2. The referee wrote: "The choice $a=i$ is also interesting when $p=2$." In this case we get from (3)

$$
H_{n}(i z)=i^{n} n ! \sum_{k=0}^{[n / 2]} \frac{2^{k}}{k !(n-2 k) !} H_{n-2 k}(z) .
$$

Remark 3. From (3) we conclude that for $n \geq 0, p \geq 1$ and $a, z \in \mathbb{C} \backslash\{0\}$ we have

$$
a^{n} \sum_{k=0}^{[n / p]}\left(1-\frac{1}{a^{p}}\right)^{k} \frac{H_{n-p k, p}(z)}{k !(n-p k) !}=z^{n} \sum_{k=0}^{[n / p]}\left(1-\frac{1}{z^{p}}\right)^{k} \frac{H_{n-p k, p}(a)}{k !(n-p k) !} .
$$

Acknowledgement. I thank the referee for helpful comments. 


\section{REFERENCES}

[1] M. Abramowitz, I.A. Stegun (eds.), Handbook of Mathematical Functions with Formulas, Graphs, and Mathematical Tables, New York, Dover, 1972.

[2] G.B. Arfken, H.J. Weber, Mathematical Methods for Physicists, Elsevier, Amsterdam, 2005.

[3] L.R. Bragg, Polynomial expansions of solutions of Cauchy problems that involve one space variable, J. Math. Anal. Appl. 214 (1997), 102-127.

[4] G.M. Habibullah, A. Shakoor, A generalization of Hermite polynomials, Int. Math. Forum 8 (2013), 701-706.

[5] G.V. Milovanović, D.S. Mitrinović, Th.M. Rassias, Topics in Polynomials: Extremal Problems, Inequalities, Zeros, World Sci., Singapore, 1994.

H. Alzer received his Ph.D. from the University of Bonn and completed his Habilitation at the University of Würzburg. He is mainly interested in special functions and inequalities.

Morsbacher Str. 10, 51545 Waldbröl, Germany

E-mail address: h.alzer@gmx.de 